\title{
Propagation characteristics of bedrock waves traveling from pre-Tertiary basement to engineering bedrock
}

\author{
Shigeo Kinoshita \\ Yokohama City University, 22-2 Seto, Kanazaw-ku, Yokohama 236-0027, Japan
}

(Received March 18, 2007; Revised September 18, 2007; Accepted September 27, 2007; Online published November 30, 2007)

\begin{abstract}
This study uses borehole array recordings to measure the propagation characteristics of bedrock waves traveling from pre-Tertiary basement (seismic bedrock) to engineering bedrock with an $S$-wave velocity of approximately $0.5-0.8 \mathrm{~km} / \mathrm{s}$. To avoid the destructive interference of surface-reflected down-going waves and incidence waves in seismic and engineering bedrock, borehole data recorded at sufficient depth levels are used in this study. This is the most important aspect of the fundamental basis of this study. The propagation characteristics, i.e., the transfer function, of bedrock motions for $S$-waves are well represented by a Butterworth-type low-pass filter model with a high corner frequency in excess of $15 \mathrm{~Hz}$ and a low decay rate of -3 power of frequency. The use of such a filter model is based on the concise representation of the transfer function from an engineering viewpoint. Simple one-dimensional ray theory with a plane wave approximation explains the characteristics of the model filter at low frequencies of less than approximately $5 \mathrm{~Hz}$; however, one-dimensional ray theory with a plane wave approximation at high frequencies in excess of $5 \mathrm{~Hz}$ requires the unusual frequency characteristics of $Q_{S}{ }^{-1}(f)$, which increases with increasing frequency, to explain the frequency characteristics of the model filter. These facts imply that the filter gain can be determined using the impedance ratio of seismic bedrock to engineering bedrock and the attenuation characteristics of the intervening media at low frequencies less than $5 \mathrm{~Hz}$. However, the cutoff frequency and decay rate of the filter must be determined from observational data.
\end{abstract}

Key words: Bedrock waves, pre-Tertiary basement, engineering bedrock.

\section{Introduction}

The use of reference sites plays an important role in seismology and earthquake engineering, particularly in the evaluation of site effects (Steidl et al., 1996; Kawase, 2003). There are two commonly used types of references sites: those installed upon or within pre-Tertiary basement, termed seismic bedrock, and those installed upon or within hard Pleistocene or Pliocene rocks, termed engineering bedrock; the choice of site depends on the purpose of the investigation. The $S$-wave velocity of seismic and engineering bedrock is greater than or equal to $2.5 \mathrm{~km} / \mathrm{s}$ and approximately $500-800 \mathrm{~m} / \mathrm{s}$ (Satoh et al., 1995), respectively. Seismic waves recorded upon or within seismic bedrock are used for studies of source characteristics (e.g., Kinoshita and Ohike, 2002a), whereas seismic waves recorded upon or within engineering bedrock, i.e., engineering bedrock waves, are used in making design code employed in the construction of important buildings and civil engineering structures. Seismic hazard analysis makes use of seismic bedrock waves or engineering bedrock waves as reference waves.

Body waves generally propagate from pre-Tertiary basement to the Pleistocene or Pliocene rocks; however, the propagation characteristics of the bedrock waves, i.e., the relation between seismic bedrock and engineering bedrock

Copyright (c) The Society of Geomagnetism and Earth, Planetary and Space Sciences (SGEPSS); The Seismological Society of Japan; The Volcanological Society of Japan; The Geodetic Society of Japan; The Japanese Society for Planetary Sciences; TERRAPUB. waves, remain unclear, as few measurements have been undertaken of the propagation characteristics. This lack of data reflects the fact that the study of bedrock waves propagating across two types of reference sites is a minor topic in seismology, even though it is important in earthquake engineering applications such as compiling local shaking-risk maps for the mitigation of earthquake disasters. In addition, the construction costs involved in developing the required observation settings for this type of study are very high; thus, significant barriers must be overcome in undertaking investigations of the propagation characteristics of bedrock waves.

The present study examines borehole array recordings obtained at the SHM and FCH stations deployed in the Kanto region, Japan. These stations were chosen because their arrays have appropriate observation settings in terms of investigations of the propagation characteristics of bedrock waves. The results obtained from these array recordings reveal that the propagation characteristics of $S$ waves are equivalent to those of a low-pass filter in the frequency domain and are partially explained by using both the impedance ratio of the seismic bedrock to the engineering bedrock and the attenuation characteristics of bedrock waves that propagate across these two bedrock types at low frequencies of less than $5 \mathrm{~Hz}$; however, this explanation, which involves one-dimensional ray theory with a plane wave approximation, remains uncertain in terms of its application at high frequencies above approximately $5 \mathrm{~Hz}$. 
(a)

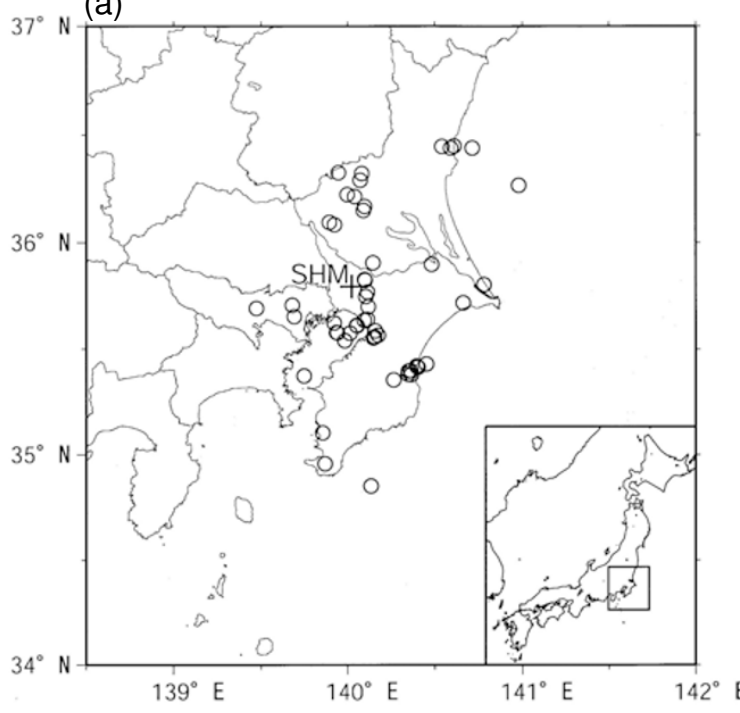

(b)

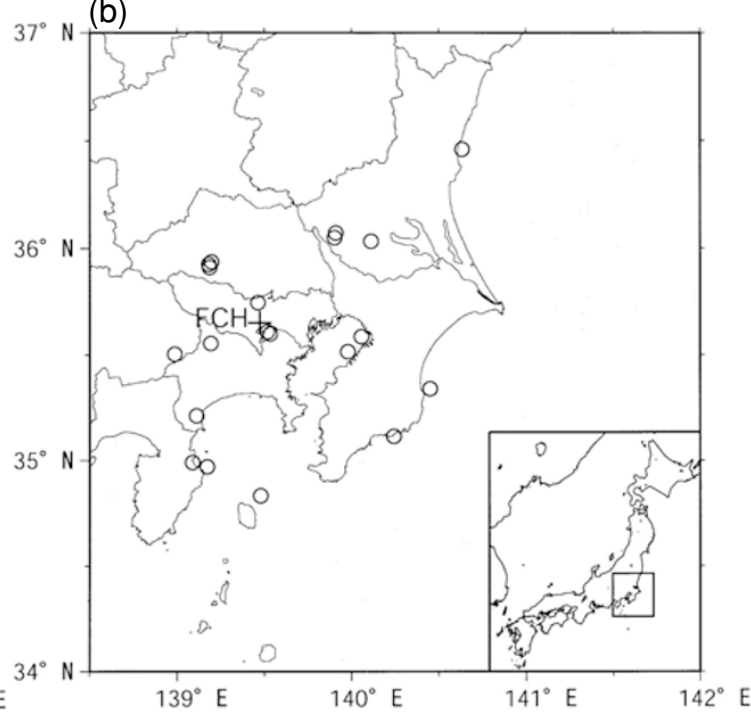

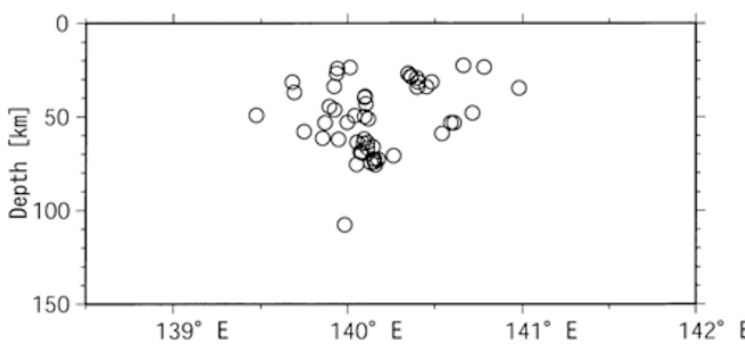

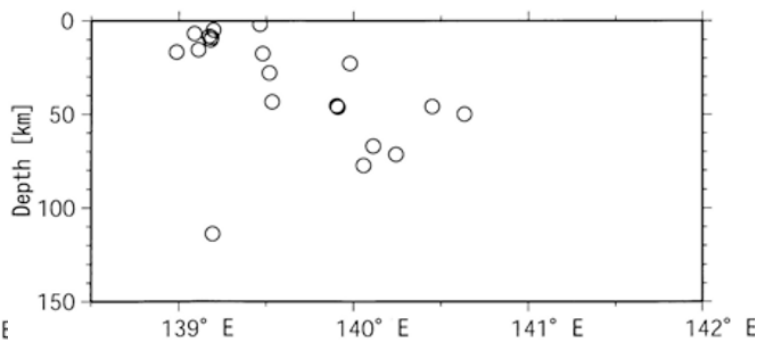

Fig. 1. (a) Map and accompanying cross-section showing the locations of events (open circles) used to estimate $G(f)$ at the SHM station (plus symbol). (b) Map and accompanying cross-section showing the locations of events (open circles) used to estimate $G(f)$ at the FCH station (plus symbol).

\section{Data}

This study is based on borehole array recordings obtained at the $\mathrm{SHM}\left(35.7934^{\circ} \mathrm{N}, 140.0238^{\circ} \mathrm{E}\right)$ and $\mathrm{FCH}$ $\left(35.6507^{\circ} \mathrm{N}, 139.4736^{\circ} \mathrm{E}\right)$ stations established by NIED (National Research Institute for Earth Science and Disaster Prevention), Japan. The station locations and hypocenters of events used in this study are shown in Fig. 1(a) for the SHM site and Fig. 1(b) for the FCH site; the total numbers of events are 54 and 20, respectively, and the ranges in magnitude are $M_{\mathrm{J}}=2.8-5.3$ and $M_{\mathrm{J}}=3.1-5.7$, where $M_{\mathrm{J}}$ represents the JMA magnitude as determined by the Japan Meteorological Agency. The observation periods employed in this study for the SHM and FCH sites are 1988-1999 and 1988-1990, respectively, and the analyzed data are restricted to direct $S$-waves.

At the SHM site, three-component sets of a negative feedback accelerometer were installed at depths of 1, 200 and $2,300 \mathrm{~m}$. These depths correspond to Pleistocene rocks (200 $\mathrm{m}$ depth) and basement rocks of pre-Tertiary crystalline schist (2,300 $\mathrm{m}$ depth), as shown in Fig. 2 (a). Vertically propagating $S$-wave velocities estimated for the Pleistocene rocks and pre-Tertiary basement using a down-hole method are 0.45 and $2.54 \mathrm{~km} / \mathrm{s}$, respectively (Ohta et al., 1978). On this basis, the seismic and engineering bedrock waves are recorded by seismometers installed at the depths of 2,300 and $200 \mathrm{~m}$, respectively. At the FCH site, the threecomponent sets of a negative feedback accelerometer are installed at depths of $1,146,500$, and 2,750 m. The deepest seismometer is located within the basement rocks of pre-
Tertiary sandstone, and the seismometer installed at $500 \mathrm{~m}$ depth is located within Plio-Pleistocene rocks, i.e., within seismic and engineering bedrock, respectively. The estimated $S$-wave velocities at the depths of 500 and $2,750 \mathrm{~m}$ are 0.78 and $2.53 \mathrm{~km} / \mathrm{s}$, respectively, as shown in Fig. 2(b) (Yamamizu et al., 1981). Thus, the seismometers installed at the depths of 2,750 and $500 \mathrm{~m}$ record seismic and engineering bedrock motions, respectively.

The same type of negative feedback accelerometer was used at both sites, with a natural frequency of approximately $450 \mathrm{~Hz}$ and a damping factor of 0.6-0.7. The flat frequency range to ground acceleration is 0 to $30 \mathrm{~Hz}(-3 \mathrm{~dB}$ point $)$, the decay rate is $-18 \mathrm{~dB} /$ octave, and the sampling rate is $100 \mathrm{~Hz}$; thus, the bedrock waves in the frequency range of 0 to $15 \mathrm{~Hz}$ are used without any correction in the frequency domain.

\section{Method}

The propagation characteristics of bedrock waves whose input and output waves are incidence waves in the seismic and engineering bedrock, respectively, are examined via the transfer function:

$$
G(f)=|Y(f) / X(f)|,
$$

where $X(f)$ and $Y(f)$ are Fourier transforms of the input and output waves, respectively, and $f$ is a frequency parameter expressed in Hz. The Fourier spectrum is calculated using the multi-taper method (Thomson, 1982), as the method 
(a)

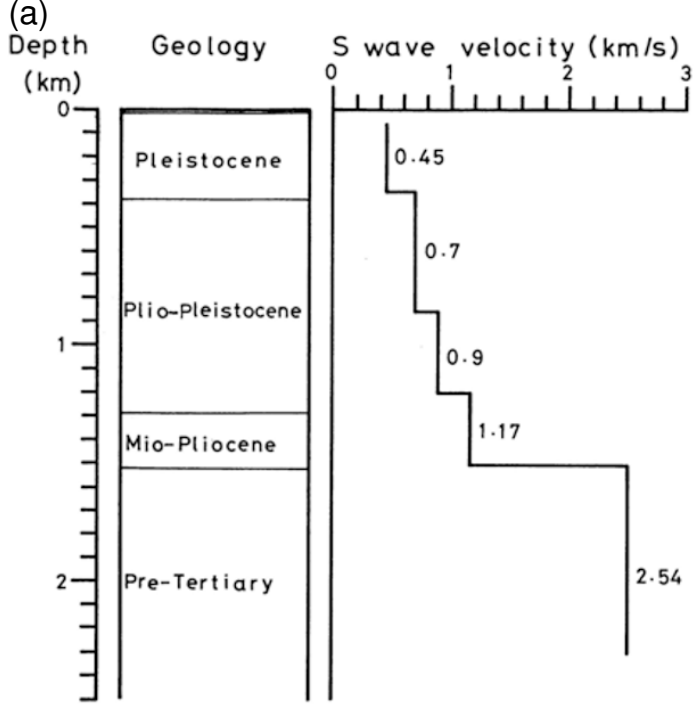

(b)

Depth Geology

S wave velocity $(\mathrm{km} / \mathrm{s})$

$(\mathrm{km})$

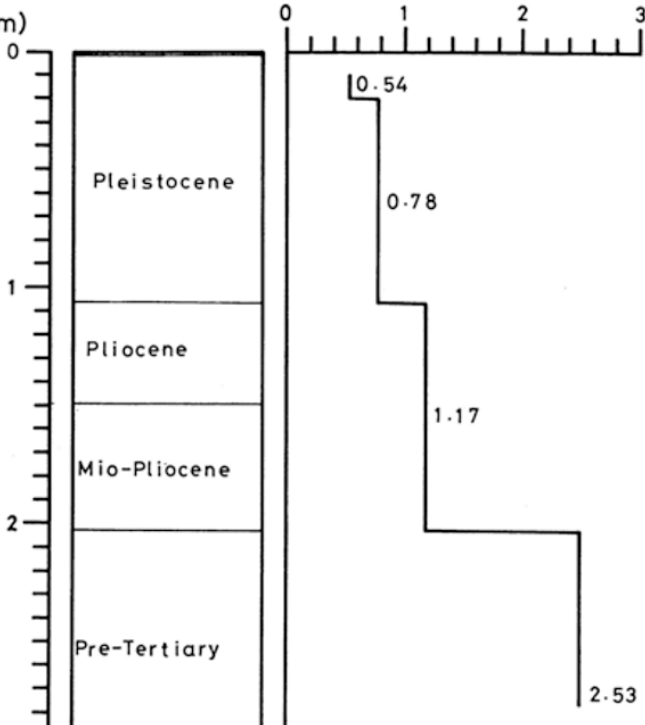

Fig. 2. (a) Velocity structure at the SHM station, as determined by Ohta et al. (1978). (b) Velocity structure at the FCH station, as determined by Yamamizu et al. (1981).

can be effectively applied to short-length data given a sufficient smoothing effect. The spectral smoothing is controlled by the time-band width $N \Delta T \cdot W$. In this study, the number of data points is $N=128$ and the sampling rate is $\Delta T=0.01 \mathrm{~s}$; thus, $N \Delta T \cdot W=4$ and $N \Delta T \cdot W=2.5$ mean that the smoothing bands are $W=3.125$ and $1.953125 \mathrm{~Hz}$, respectively. Furthermore, as a tentative model of $G(f)$, the following Butterworth-type low-pass filter is applied to the mean values of $G(f)$ estimated via relation (1) using a Gauss-Newton nonlinear least squares method:

$$
G(f)=G_{o} /\left[1+\left(f / f_{c}\right)^{n}\right]
$$

The model is characterized by a gain of $G_{o}$, a corner frequency of $f_{c}$, and a power of $n$. The physical meaning of $G_{o}$ is discussed below.

The estimation procedure for $G(f)$ is illustrated using the following example. The array recordings depicted in Fig. 3 were obtained at the FCH site for an earthquake that occurred on 25 February 1990. These transverse components are displacement waves found by integrating the original acceleration seismograms in order to illustrate the propagation of direct $S$-waves within a sedimentary deposit. The direct $S$-wave at the depth of 2,750 m (upper figure) in the seismic bedrock propagates toward the ground surface (lower figure); subsequently, the surface-reflected $S$-wave travels downward. This type of geological structure, composed of the basement and an overlying sedimentary deposit, is termed a "sedimentary layer-basement system" (Kinoshita and Ohike, 2006). The direct $S$-wave approaches the surface within the system with increasing amplitude. Its surface-reflected $S$-wave, which has a similar waveform to that of the upward $S$-wave at each depth level except for the deepest level in this case, travels downward toward the engineering bedrock at $500 \mathrm{~m}$ depth. Hence, it is possible to identify the incidence $S$-phase on the seismo-

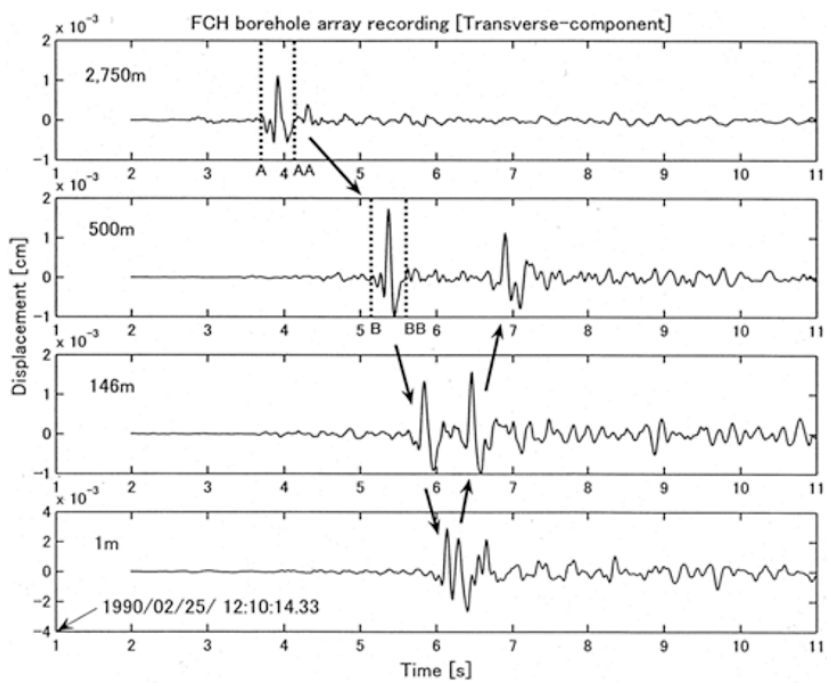

Fig. 3. Transverse-component array seismograms recorded at the FCH site for the event that occurred on 25 February 1990, including array seismograms that illustrate the direct $S$-phase traveling within the sedimentary layer-basement system. $G(f)$ is estimated using the direct $S$-phase represented by the dashed lines labeled "A" and "AA" on the seismogram at $2,750 \mathrm{~m}$ depth and that represented by the dashed lines labeled "B" and "BB" on the seismogram at $500 \mathrm{~m}$ depth.

grams recorded at depths of 2,750 and $500 \mathrm{~m}$. First, the direct $S$-phase in the seismic bedrock is determined visually, as marked by two dashed lines labeled "A" and "AA" in Fig. 3. This phase is a reference phase; that is, an input wave for $G(f)$. Next, by moving this reference phase on the seismogram recorded at the depth of $500 \mathrm{~m}$ in the engineering bedrock, the cross-correlation functions between the reference wave and the bedrock waves at $500 \mathrm{~m}$ depth, that is, output waves, are calculated. The delay time $t^{*}$ at which the calculated cross-correlation function has its max- 
imum is then determined, along with the output data window, as marked by two dashed lines labeled "B" and "BB" in Fig. 3. Applying the multi-taper method to these two data windows, $X(f)$ and $Y(f)$ are calculated, followed by $G(f)$.

To avoid the destructive interference of surface-reflected down-going waves and incidence waves at the depth of shallow boreholes (200 and $500 \mathrm{~m}$ depths at the SHM and FCH sites, respectively) drilled into engineering bedrock, the data length of the reference phase must be less than the two-way time, $2 t_{o}^{*}$, of $S$-waves traveling between the surface and the depth level of shallow borehole; Fig. 3 confirms that this requirement is satisfied. However, for some events for which the data length of the reference phase is longer than $2 t_{o}^{*}$, the window length of the reference phase to which Fourier analysis is applied is truncated with a length of $2 t_{1}^{*}\left(<2 t_{o}^{*}\right)$ from the onset point of the reference wave. The values of $2 t_{1}^{*}$ used in this study are 0.9 and $1.28 \mathrm{~s}$ at the SHM and FCH sites, respectively, as determined according to the theoretical two-way times, $2 t_{o}^{*}=1.03$ and $1.64 \mathrm{~s}$, which are calculated using the velocity structures at the SHM and FCH sites (Yamamizu et al., 1981, 1983). The transfer function $G(f)$ is estimated in the following section using two different data lengths: one being the length of the direct $S$-phase and the other being the fixed data length of $2 t_{1}^{*}$. This approach is taken to investigate the influence of data length on estimates of $G(f)$.

\section{Results}

First, the $G(f)$ estimated using the data length of direct $S$-phase at the SHM site is shown in Fig. 4, as obtained by applying a multi-taper method with $N \Delta T \cdot W=4$ to the array recordings obtained from the events shown in Fig. 1(a). The mean values of $G(f)$ are represented by solid circles in Fig. 4, and the one-standard-error range is indicated by dashed lines. The result of fitting relation (2) to the mean estimates is shown by the solid line in Fig. 4, yielding the following function:

$$
G(f)=\frac{(2.48 \pm 0.06)}{1+[f /(17.7 \pm 1.1)]^{2.72 \pm 0.55}} .
$$

The accuracy of the fit of the tentative model given by relation (2) is so height that this model is used hereafter. These estimation data comprise combined transverse and radial components because the difference in $G(f)$ estimated using transverse- and radial-component data separately is insignificant. Figure 5 shows the results obtained from the same dataset using $N \Delta T \cdot W=2.5$; the best-fit model (solid line in Fig. 5) is given by

$$
G(f)=\frac{(2.52 \pm 0.10)}{1+[f /(17.4 \pm 1.5)]^{2.66 \pm 0.78}} .
$$

Given that the differences in the estimated results obtained using $N \Delta T \cdot W=2.5$ and $N \Delta T \cdot W=4$ are minor, the estimates of $G(f)$ obtained using the multi-taper method $N \Delta T \cdot W=4$ are discussed hereafter.

Next, Fig. 6 shows the results estimated for the FCH site from the array recordings for the events shown in Fig. 1(b). The solid circles and dashed lines in the figure represent the

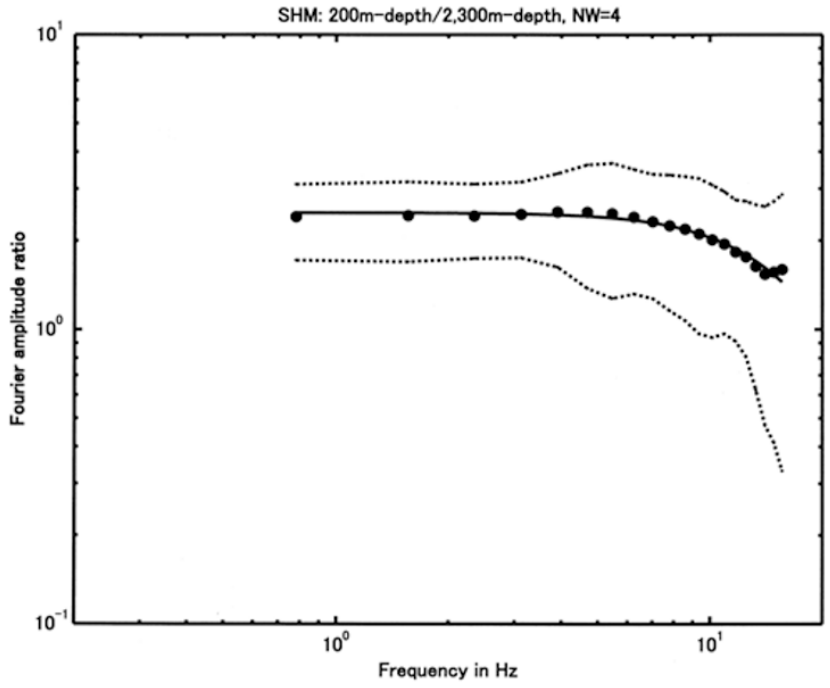

Fig. 4. Estimates of $G(f)$ at the SHM site (solid circles) obtained using a multi-taper method with a time-bandwidth of 4 . The solid line represents the best-fit model for $G(f)$.

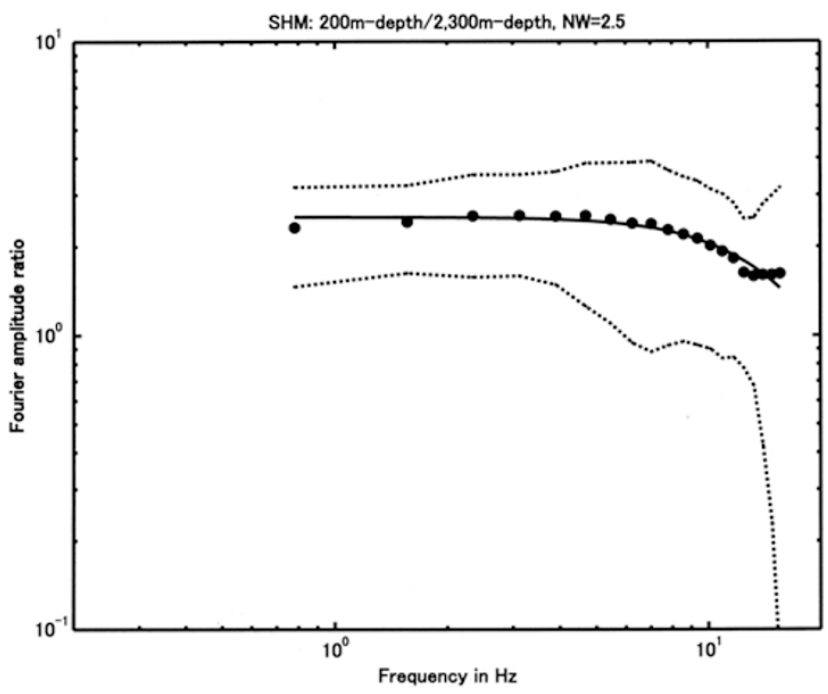

Fig. 5. Estimates of $G(f)$ at the SHM site (solid circles) obtained using a multi-taper method with a time-bandwidth of 2.5. The solid line represents the best-fit model for $G(f)$.

mean estimates of $G(f)$ and the one-standard-error range, respectively. The best-fit model for relation (2) is shown by the solid line in Fig. 6, and is given by

$$
G(f)=\frac{(1.95 \pm 0.07)}{1+[f /(15.1 \pm 0.8)]^{3.24 \pm 0.79}} .
$$

The fact that the above results of $G(f)$ obtained at the SHM and $\mathrm{FCH}$ sites have the frequency characteristics of a lowpass filter with a high cutoff frequency in excess of $15 \mathrm{~Hz}$. This means that in estimating the source characteristics at high frequencies using the spectral inversion method for a reference site located upon or within engineering bedrock, frequency compensation may be required for the reference wave, especially in estimating source-controlled $f_{\max }(\mathrm{Ki}$ noshita, 1992).

Finally, the influence of data length on the estimates of $G(f)$ is examined. The fixed data lengths of 0.9 and $1.28 \mathrm{~s}$ 


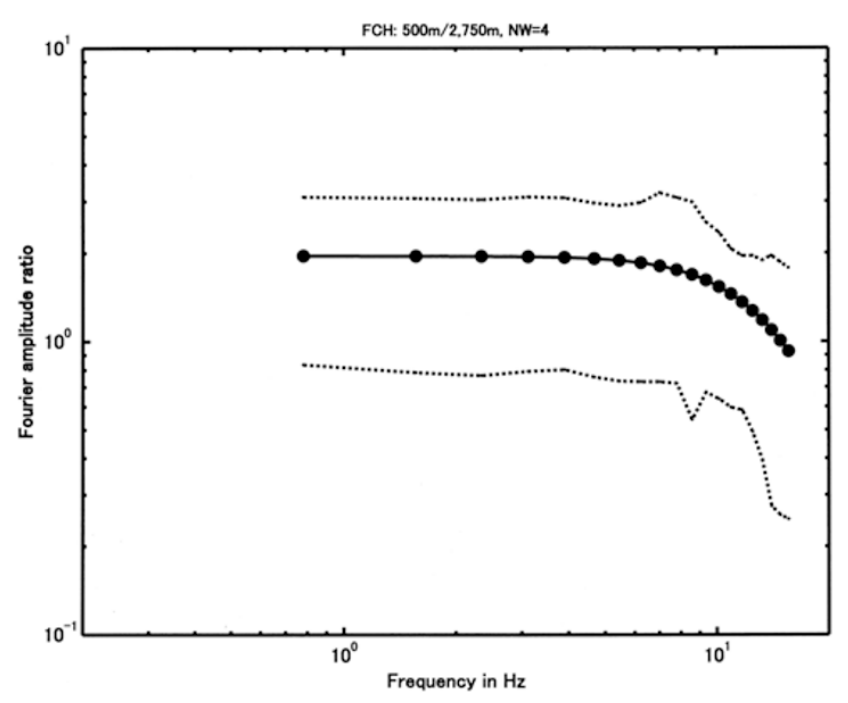

Fig. 6. Estimates of $G(f)$ at the $\mathrm{FCH}$ site (solid circles) obtained using a multi-taper method with a time-bandwidth of 4 . The solid line represents the best-fit model for $G(f)$.

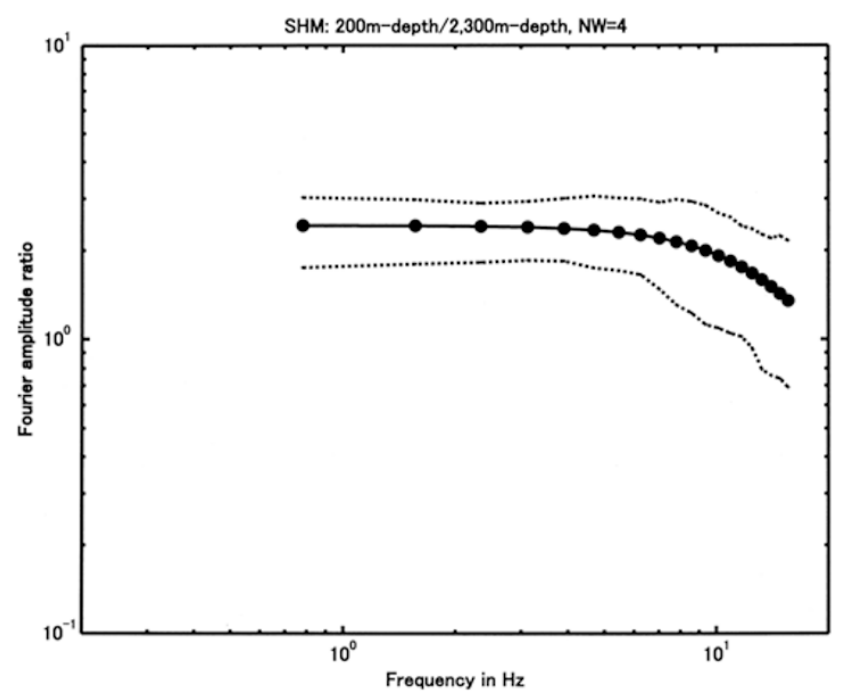

Fig. 7. Estimates of $G(f)$ at the SHM site (solid circles) obtained using a multi-taper method with a time-bandwidth of 4 and data length of $0.9 \mathrm{~s}$. The solid line represents the best-fit model for $G(f)$.

discussed in the previous section are used for the recordings obtained at the SHM and FCH sites, respectively. For the SHM site, the estimated mean values of $G(f)$ are shown by solid circles in Fig. 7; the best-fit model represented by the solid line is given by

$$
G(f)=\frac{(2.43 \pm 0.05)}{1+[f /(17.0 \pm 0.8)]^{2.55 \pm 0.37}}
$$

Similarly, estimates of $G(f)$ for the $\mathrm{FCH}$ site are represented by solid circles in Fig. 8. The best-fit model represented by the solid line in Fig. 8 is given by

$$
G(f)=\frac{(1.88 \pm 0.03)}{1+[f /(15.8 \pm 0.5)]^{3.20 \pm 0.45}}
$$

The difference between these results and the estimates obtained from direct phase data is insignificant. This means

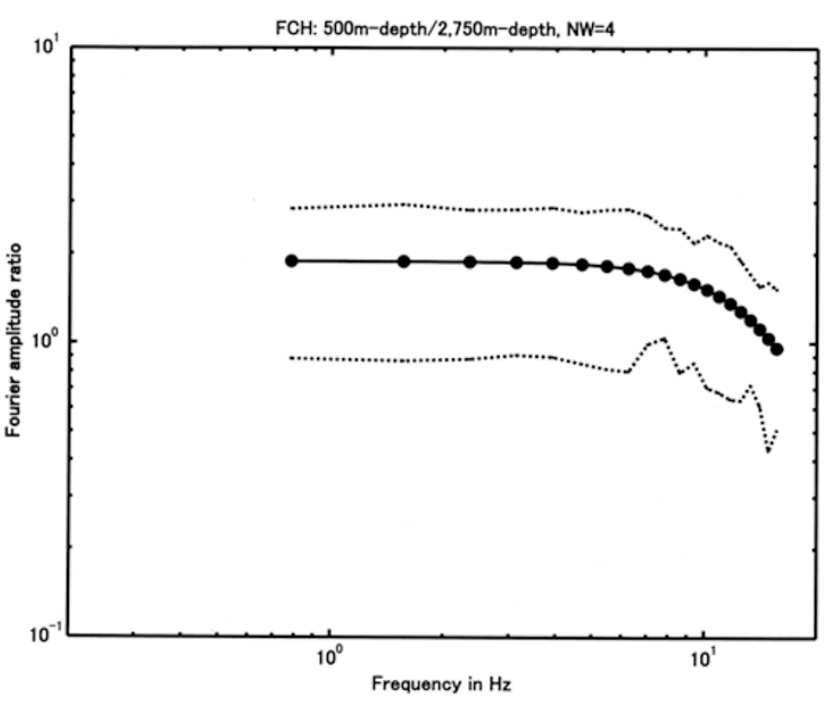

Fig. 8. Estimates of $G(f)$ at the FCH site (solid circles) obtained using a multi-taper method with a time-bandwidth of 4 and data length of $1.28 \mathrm{~s}$. The solid line represents the best-fit model for $G(f)$.

that the influence of coda energy on the estimation of $G(f)$ is minor and that the selection of array recordings used for the estimation of $G(f)$ in this study are adequate.

\section{Discussion}

The estimated $G(f)$, as shown in Figs. 4-8, has the frequency characteristics of the low-pass filter given by relation (2). This discussion examines the extent to which simple one-dimensional ray theory with a plane wave approximation is applicable to the resultant $G(f)$. The discussion is narrowed down to the result shown in Fig. 4, as this result was derived from a large amount of data at the SHM site compared with the relatively small amount of data used for the $\mathrm{FCH}$ site. The transfer function $G(f)$, whose input and output are given by bedrock waves in seismic and engineering bedrock, respectively, can be written as follows according to one-dimensional ray theory with a plane wave approximation:

$$
\begin{aligned}
& G(f)=G_{o} \cdot \exp \left[-\omega t^{*} / 2 Q_{S}(f)\right], \\
& G_{o}=\sqrt{\left(\rho V_{S}\right)_{\mathrm{SB}} /\left(\rho V_{S}\right)_{\mathrm{EB}}},
\end{aligned}
$$

where $\left(\rho V_{S}\right)_{\mathrm{SB}}$ and $\left(\rho V_{S}\right)_{\mathrm{EB}}$ are the $S$-wave impedance of seismic and engineering bedrock, respectively. $Q_{S}(f)$ and $t^{*}$ represent the frequency-dependent value of $Q$ in the media between the two depth levels at which seismometers are installed in the seismic and engineering bedrock and the one-way time of $S$-waves traveling through the media, respectively. The $S$-wave impedance was measured at the time of the construction of the deepest borehole (Ohta et al., 1978; Yamamizu et al., 1981), and the calculated $G_{o}$ is 2.76 at the SHM site and 2.01 at the FCH site. The oneway time $t^{*}$ is estimated in this study for the time when the data windows used in estimating $G(f)$ were determined. $t^{*}=1.87 \pm 0.04 \mathrm{~s}$ and $t^{*}=1.51 \pm 0.06 \mathrm{~s}$ are the mean value and one-standard-error range estimated at the SHM and FCH sites, respectively.

Because relation (3) indicates that the frequency characteristics of $G(f)$ are controlled by $1 / Q_{S}(f)$, the exam- 


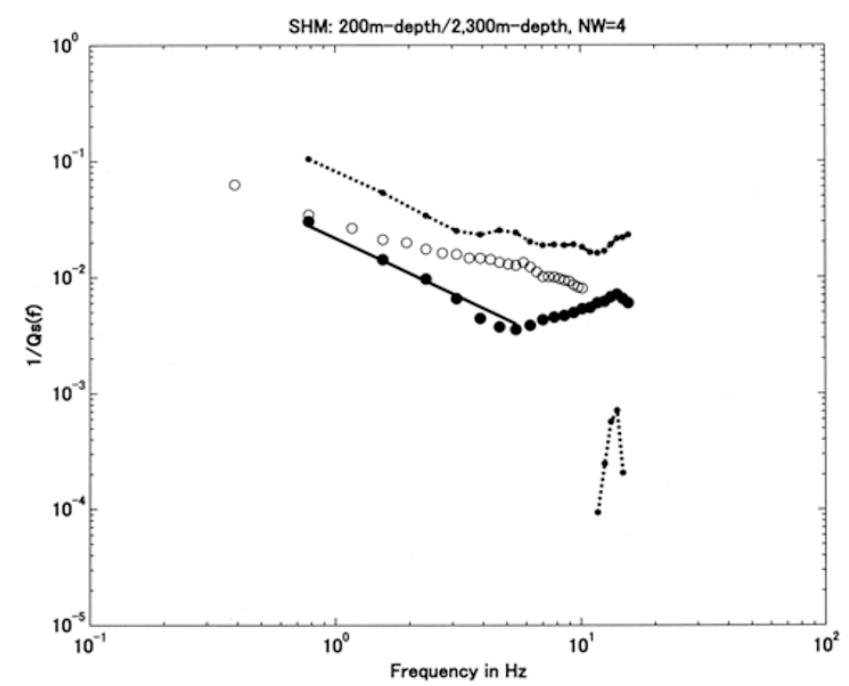

Fig. 9. Estimate of $Q_{S}^{-1}(f)$ (solid circles) and the one-standard-error range (dashed lines) at the SHM site. Open circles represent the estimates of $Q_{S}^{-1}(f)$ at the SHM site for $S$-waves in a sedimentary layer-basement system, as determined by Kinoshita (2007).

ination of relation (3) focuses on whether the estimated $1 / Q_{S}(f)$ is empirically reasonable. $1 / Q_{S}(f)$ is estimated using the following relation:

$$
\hat{Q}_{S}^{-1}(f)=\frac{1}{\pi f t^{*}} \cdot \ln \left[G_{o} / \hat{G}(f)\right],
$$

where $\hat{G}(f)$ is an estimate of $G(f)$ (the mean values shown by solid circles in Fig. 4). The solid circles in Fig. 9 represent the mean estimates of $1 / \hat{Q}_{S}(f)$ obtained at the SHM site.

Thus, the frequency characteristics $\hat{G}(f)$ of the Butterworth-type filter obtained in this study are calculated using relation (3) and $\hat{Q}_{S}^{-1}(f)$ shown by solid circles in Fig. 9. The estimates of $\hat{Q}_{S}^{-1}(f)$ represent the frequency structure within which $\hat{Q}_{S}^{-1}(f)$ decreases with increasing frequency for frequencies up to approximately $5 \mathrm{~Hz}$, and then increases with increasing frequency for frequencies above $5 \mathrm{~Hz}$. Hence, the discussion on the relation between $\hat{G}(f)$ and $\hat{Q}_{S}^{-1}(f)$ is divided into two frequency ranges, with a boundary frequency of approximately $5 \mathrm{~Hz}$. The low-frequency range in which $\hat{G}(f)$ has a flat gain corresponds to the frequencies at which $\hat{Q}_{S}^{-1}(f)$ decreases with increasing frequency. Such a frequency structure of $\hat{Q}_{S}^{-1}(f)$ for a sedimentary layer-basement system has been commonly reported in previous studies (e.g., Kinoshita and Ohike, 2002b). In this frequency range, the flat level $\hat{G}_{o}=0.88 G_{o}$ is obtained using $\hat{Q}_{S}^{-1}(f=1 \mathrm{~Hz}) \equiv$ $Q_{o}{ }^{-1}=0.0217$, assuming $\hat{Q}_{S}^{-1}(f)=Q_{o}{ }^{-1} f^{-1}$ shown by the solid line in Fig. 9. Similarly, $\hat{G}_{o}=0.94 G_{o}$ is obtained using $Q_{o}{ }^{-1}=0.0130$ at the $\mathrm{FCH}$ site. The value of $Q_{o}{ }^{-1}$ is dependent on the $S$-wave velocity of engineering bedrock, though this is derived from only two datasets; $Q_{o}{ }^{-1}$ decreases with increasing $S$-wave velocity of engineering bedrock. Therefore, it is reasonable to draw the conclusion that one-dimensional ray theory with a plane wave approximation represented by relation (3) is applicable to the low-pass characteristics of $\hat{G}(f)$ for frequencies less than $5 \mathrm{~Hz}$ when using a frequency-dependent $\hat{Q}_{S}^{-1}(f)$ $\left(=Q_{o}{ }^{-1} f^{-1}\right)$.

In contrast, for the high frequency range above $5 \mathrm{~Hz}$, one-dimensional ray theory with a plane wave approximation must use $Q_{S}{ }^{-1}(f)$, which increases with increasing frequency (solid circles in Fig. 9), in order to explain the high-frequency decay part of $\hat{G}(f)$. However, it is difficult to find observational proof that $Q_{S}^{-1}(f)$ increases with increasing frequency at high frequencies above $5 \mathrm{~Hz}$. The measurement of $Q_{S}^{-1}(f)$ for thick sediments or in seismogenic zones shows that $Q_{S}^{-1}(f)$ decreases with increasing frequency or is frequency-independent, as described by Abercrombie (1998). For example, the open circles in Fig. 9 are estimated $\hat{Q}_{S}^{-1}(f)$ for media between the surface and the $2300 \mathrm{~m}$ depth level; i.e., a sedimentary layerbasement system at the SHM site (Kinoshita, 2007). The $\hat{Q}_{S}^{-1}(f)$ shown by open circles is affected by a soft surface layer with a thickness of $200 \mathrm{~m}$ compared with the $\hat{Q}_{S}^{-1}(f)$ shown by solid circles, which are obtained for media between 200 and $2300 \mathrm{~m}$ depths. Hence, it is reasonable that the frequency-dependent mean values of $\hat{Q}_{S}^{-1}(f)$ shown by solid circles are slightly small compared with the mean values of $\hat{Q}_{S}^{-1}(f)$ shown by open circles, as obtained for the sedimentary layer-basement system. However, $\hat{Q}_{S}^{-1}(f)$ shown by open circles decreases with increasing frequency up to $10 \mathrm{~Hz}$, and thus is concordant with many observational facts (e.g., Abercrombie, 1998). $\hat{Q}_{S}^{-1}(f)$ shown by solid circles increases with increasing frequency, and is clearly different from the frequency structure of $\hat{Q}_{S}^{-1}(f)$, shown by open circles, for frequencies higher than $5 \mathrm{~Hz}$. Taking into account the unusual structure of $Q_{S}^{-1}(f)$, as shown by solid circles in Fig. 9, it is therefore natural to directly determine the filter parameters $f_{c}$ and $n$ in relation (2) used for the evaluation of $G(f)$ from observational data instead of using relation (3).

Finally, the flat level of $G(f)$ defined by relation (3) for frequencies less than $5 \mathrm{~Hz}$ is controlled by the one-way time $t^{*}$, which is influenced by the incidence angle of the reference wave in seismic bedrock. The flat level range of $2.43 \pm 0.01$ is obtained from estimated $t^{*}=1.87 \pm 0.04$ s using relation (3) and $Q_{o}{ }^{-1}=0.0217$. The minimum and maximum values of $t^{*}$ estimated for the dataset used in this study are 1.81 and $1.94 \mathrm{~s}$, respectively, and the corresponding flat levels are 2.44 and 2.42 , respectively. Similarly, at the $\mathrm{FCH}$ site the flat level range of $G(f)$ is $1.89 \pm 0.01$ according to the estimates of $t^{*}=1.51 \pm 0.06 \mathrm{~s}$ and $Q_{o}{ }^{-1}=0.0130$. Flat levels of 1.90 and 1.88 are calculated for the minimum and maximum values of estimated $t^{*}$; i.e., 1.40 and $1.62 \mathrm{~s}$, respectively. These facts demonstrate that the contribution of one-way time $t^{*}$, as estimated from the datasets used in this study, to the flat level of $G(f)$ defined by relation (3) is negligible.

\section{Conclusions}

Direct measurements derived from borehole array recordings reveal the frequency characteristics of bedrock waves (direct $S$-waves) that propagate from seismic bedrock to engineering bedrock. When the data are free from destructive interference between surface-reflected down-going waves and upward incidence waves, the ob- 
tained results are straightforward, and can be summarized as follows.

(1) The transfer function whose input and output waves are incidence $S$-waves recorded in the pre-Tertiary basement and those recorded in hard rock sites (with $S$-wave velocities of approximately $0.5-0.8 \mathrm{~km} / \mathrm{s}$ ), respectively, has the frequency characteristics of the following Butterworth-type low-pass filter:

$$
G(f)=G_{o} /\left[1+\left(f / f_{c}\right)^{n}\right]
$$

where the corner frequency $f_{c} \sim 15-18 \mathrm{~Hz}$ and the power $n$ is approximately 3 , as determined empirically from observational data.

(2) The estimated transfer function has the characteristic of flat gain for frequencies less than $5 \mathrm{~Hz}$. This gain is explained using one-dimensional ray theory with a plane wave approximation represented by relation (3); it is calculated using the square root of the impedance ratio of seismic bedrock to engineering bedrock and $t^{*} / Q_{S}(f=1 \mathrm{~Hz})=t^{*} / Q_{o}$ for $S$-waves within the intervening media. The estimated $Q_{o}{ }^{-1}$ are 0.0217 and 0.0130 for the SHM and FCH sites, respectively, while the estimated mean values of one-way time $t^{*}$ are 1.87 and $1.51 \mathrm{~s}$.

Acknowledgments. The author is grateful to two anonymous reviewers for carefully reviewing the manuscript and providing valuable comments.

\section{References}

Abercrombie, R. E., A summary of attenuation measurements from borehole recordings of earthquakes: the $10 \mathrm{~Hz}$ transition problem, Pure Appl. Geophys., 153, 475-487, 1998.

Kawase, H., Site effects on strong ground motions, in International Handbook of Earthquake \& Engineering seismology, 1013-1030, Academic Press, 2003.

Kinoshita, S., Local characteristics of $f_{\max }$ of bedrock motion in the Tokyo metropolitan area, Japan, J. Phys. Earth, 40, 487-515, 1992.

Kinoshita, S., Deep-borehole measured Qp and Qs attenuation for two Kanto sedimentary deposit sites, Bull. Seis. Soc. Am., 2007 (in press).

Kinoshita, S. and M. Ohike, Scaling relations of earthquakes that occurred in the upper part of the Philippine sea plate beneath the Kanto region, Japan, estimated by means of borehole recordings, Bull. Seis. Soc. Am., 92, 611-624, 2002a.

Kinoshita, S. and M. Ohike, Attenuation characteristics of S-waves in a sedimentary layer-basement system in the Kanto region, Japan, for a frequency range of 0.5 to $16 \mathrm{~Hz}, \mathrm{Zisin}, \mathbf{5 5}, 19-31,2002 \mathrm{~b}$ (in Japanese).

Kinoshita, S. and M. Ohike, Coherency characteristics of body waves traveling in a sedimentary layer-basement system in the Kanto region, Japan, Bull. Seis. Soc. Am., 96, 165-175, 2006.

Ohta, Y., N. Goto, K. Shiono, H. Takahashi, F. Yamamizu, and S. Kurihara, Shear wave velocities in deep soil deposits, Zisin, 31, 299-308, 1978.

Satoh, T., T. Sato, and H. Kawase, Evaluation of local site effects and their removal from borehole records observed in the Sendai Region, Japan, Bull. Seis. Soc. Am., 85, 1770-1789, 1995.

Steidl, J. H., A. G. Tumarkin, and R. J. Archuleta, What is a reference site?, Bull. Seis. Soc. Am., 86, 1733-1748, 1996.

Thomson, D. J., Spectrum estimation and harmonic analysis, Proc. of IEEE, 70, 1055-1096, 1982.

Yamamizu, F., H. Takahashi, N. Goto, and Y. Ohta, Shear waves velocities in deep deposits, Zisin, 34, 465-479, 1981 (in Japanese).

Yamamizu, F., N. Goto, Y. Ohta, and H. Takahashi, Attenuation of shear waves in deep soil deposits as revealed by down-hole measurement in the 2300 meter-borehole of the Shimosa observatory, Japan, J. Phys. Earth, 31, 139-157, 1983.

S. Kinoshita (e-mail: kkk001@yokohama-cu.ac.jp) 